Research Paper

\title{
Can Occult Cystobiliary Fistulas in Hepatic Hydatid Disease Be Predicted Beiore Surgery?
}

\author{
Kemal Atahan ${ }^{\bowtie}$, Hakan Küpeli, Mehmet Deniz, Serhat Gür, Atilla Çökmez, Ercüment Tarcan \\ Atatürk Educational and Research Hospital 1st Surgical Department, İzmir, Turkey
}

Corresponding author: Kemal Atahan, 6342 sok. No:44 Ayşe Kaya 2 Apt. Kat:3, Daire:6 35540 Bostanlı/İzmir/TURKEY. Phone: +905324126805; Fax: +902322445624 ; e-mail: kemalatahan @yahoo.com.tr.

(C) Ivyspring International Publisher. This is an open-access article distributed under the terms of the Creative Commons License (http://creativecommons.org/ licenses/by-nc-nd/3.0/). Reproduction is permitted for personal, noncommercial use, provided that the article is in whole, unmodified, and properly cited.

Received: 2011.03.23; Accepted: 2011.05.11; Published: 2011.05.19

\begin{abstract}
Background: Biliary fistulas because of the cystobiliary communication is the most frequent and undesirable postoperative complication of hepatic hydatid surgery. We aimed to identify the predicting factors of the occult cystobiliary communication in this study.

Methods: The patients who underwent surgical treatment for hepatic hydatid disease between 2003 and 2008 were reviewed retrospectively. The patients who had jaundice history, preoperative high total bilirubin and direct bilirubin levels, dilated bile duct in preoperative radiologic imagings were not included the study. Patients were divided into two groups: group A; without postoperative biliary fistula, group B; with biliary fistula. The two groups were compared according to preoperative descriptive findings, cystic specialties, and laboratory findings.

Results: There were 53 patients and 15 patients in groupA and groupB, respectively. The 20 $(37.7 \%)$ of 53 patients were male in group $A$ and the $10(66.7 \%)$ patients were male in group B $(p<0.05)$. The age, number of cysts, Garbi scores of cysts, the rate of recurrent cysts, the level of preoperative bilirubine, alkalene phosphatase, and transaminases were similar in both groups $(p>0.05)$. GGT was significantly different between two groups $(p<0.05)$. The cystotomy + drainage, cystotomy + omentopexy, and intracystic biliary suture rates were similar in both groups. Postoperative non biliary complications were determined in 4 (7.5\%) patients in group $A$ and 7 patients $(46.7 \%)$ in group $B(p<0.05)$. Hospital stay was longer in group $B$ significantly $(p<0.05)$.

Conclusions: In conclusion, GGT as a labaratory test for predicting occult CBC preoperatively have been shown to be useful in the clinical practice. However, larger prospective studies are needed on this subject. Occult cysto-biliary fistulas can only be exposed during surgery when suspected by a surgeon. If occult $C B C$ is found, the opening in the biliary system should be sutured with absorbable material, with or without cystic duct drainage. If no biliary opening is found, cystic duct drainage may be performed if preoperative factors predict the presence of $\mathrm{CBC}$. As the development of external biliary fistulas increases the morbidity and the hospitalization period, novel surgical methods to prevent the development of bile fistulas are required in such patients.
\end{abstract}

Key words: Biliary fistulas, cystobiliary communication, hepatic hydatid disease, cyst, surgery

\section{Background}

Hydatid Disease is a parasitic infection which caused by echinicocus granulosus (1). It is an endemic

disease in Turkey (2-4). In $50-70 \%$ of the patients, the liver is infected by the disease (5). Hepatic hydatid 
disease (HHD) has some complications but the most common complication is cystobiliary comunication (CBC) $(6,7)$. The rate of CBC in the literature is $13-37 \%$ $(8,9)$. Two kind of $C B C$ has been presented as frank and occult (10). All of these complications are increasing the mortality and morbidity rate and extending the hospital stay day (11). In this study we aimed to identify the predicting factors of occult CBC.

\section{Methods}

The patients who were performed on surgical treatment for HHD consecutively in the First Surgical department of İzmir Ataturk Training and Research Hospital between January 2003 and December 2007 have been reviewed retrospectively. In this period 85 patients underwent surgical treatment for HHD. The diagnosis of HHD was confirmed by US and/or CT in all of the patients and it was confirmed that there was no other cyst in the other solid organs by the same techniques. We used the hospital archives for the study. The physical examinations, imaging findings, laboratory findings of all the patients were documented. The patients who had jaundice attack before hospitalization, with total bilirubin value was higher than $2.0 \mathrm{mg} / \mathrm{dl}$ and direct bilirubin level was higher than $1.5 \mathrm{mg} / \mathrm{dl}$ were excluded from the study. On the other hand the patients who had common bile duct dilatation (more than $10 \mathrm{~mm}$ ) or intrahepatic biliary dilatation in US or CT or MR were also excluded. These patients have been accepted as frank CBC. The patients, who have not frank $\mathrm{CBC}$, were performed external drainage with or without omentoplasty. In our external drainage procedure we input 2 drains in all cysts and before the inputting we explore for biliary yielding in the cyst and if we find a site of biliary coloring we stitch there with unabsorbable sutures.

Included patients were divided into two groups. The patients who had no biliary fistulisation after surgery was in group A and who had biliary fistulisation were in group B. Two groups were compared according to the age, sex, number of the cyst, site of the cyst, preoperative laboratory findings (total bilirubin, direct bilirubin, ALP, AST, ALT, GGT, white blood cells, eosinophills), Garby score of the cysts, type of the operation, extrabiliary complications and postoperative hospitality days. Mann-Whitney U and Chi-square tests were used for statistical analysis and lower than $0.05 \mathrm{p}$ value has been accepted statistically significant.

\section{Results}

Totally 85 patients have been operated because of HHD in our clinic between 2002-2007. Twelve of 85 patients had frank CBC and 5 patients' files were not enough for reviewing. Therefore 17 patients have been excluded from the study. The $55.9 \%$ percent of the remaining 68 patients which included in the study were female and the overall age was 41.1 years. Total cyst number was 80 in 68 patients. All of the patients had complaint of abdominal pain. On physical examination a right upper quadrant mass was detected in 10 patients (14\%); the other physical examination findings were normal. Fifteen of the 68 patients were complicated with external biliary fistula (22.1\%). In our study there were no patients with biliary peritonitis or biliary abscess. Then 53 patients have been included in group A and 15 patients included in group $\mathrm{B}$.

The mean age of the patients in group A was $41.2 \pm 14.2$ years and in group B was $41.1 \pm 16.4$ years. The number of male patients were $20(37.7 \%)$ in group A but 10 patients $(66.7 \%)$ in group B. This difference was significant statistically $(\mathrm{p}<0.05)$ (Table 1$)$. In group A 23 patients (43.3\%) applied to the hospital from rural region and in group B this number was 6 patients $(40.0 \%)$. There was no statistically difference according to the origin of the patients $(p>0.05)$. In group A $90.6 \%$ of the patients had primer cysts and in group B the rate of the patients who had primer cysts was $86.7 \%$. The cysts were located in the right lobe in $37(69.8 \%)$, in the left lobe in $6(11.3 \%)$, and in the right+left lobe in $10(18.9 \%)$ patients in Group A. The cyst locations were right, left and right+left in 12 $(80.0 \%), 1(6.7 \%), 2(13.3 \%)$ patients respectively in group B. All characteristics of the cysts were not different statistically between two groups ( $p>0.05)$.

The laboratory findings were not statistically different between two groups except GGT values $(p>0.05)$ (Table 2). GGT was significantly different between two groups $(p<0.05)$.

We performed external drainage to 29 patients $(54.7 \%)$, external drainage+omentopxy to 23 patients (43.4\%), and cystectomy to $1(1.9 \%)$ patient in group A. External drainage was performed to 6 patients $(40 \%)$ and external drainage+omentopexy was performed to 9 patients $(60 \%)$ in group B. There was no patient who was performed cystectomy in group B. The operations were similar between two groups $(p>0.05)$. In group A, biliary communication was determined in 14 patients (26.4\%) and intracystic suture ligation was performed on the site of biliary leakage. Intracystic suture ligation was performed on 5 patients $(33.3 \%)$ in group B. There was no difference between two groups ( $p>0.05)$.

Postoperative non biliary complications visualized in $4(7.5 \%)$ patients in group A. All of these complications were wound infection and were treated by antibiotherapy and dressing easily. In group B 
there were 7 patients $(46.7 \%)$ with nonbiliary complications. Six patients had wound infection and were treated with antibiotherapy and dressing. One patient had lung complication. This patient was treated by ventilation and antibiotherapy. The complication rate was significantly higher in group B than group A $(p<0.05)$. There was no mortality in both groups.

Table I: Descriptive characters of the patients

\begin{tabular}{lll}
\hline & Group A (n=53) & Group B $(\mathrm{n}=15)$ \\
\hline Age (Years) & $41.1(11-73)$ & $41.2(19-64)$ \\
Sex $(\mathrm{M} / \mathrm{F})^{*}$ & $20 / 33$ & $10 / 5$ \\
Size $(\mathrm{mm})$ & $83.4(55-400)$ & $73.6(48-300)$ \\
Number of the cyst & $1.3(1-2)$ & $1.4(1-2)$ \\
Gharby score & $2.3(2-3)$ & $2.5(2-3)$ \\
\hline
\end{tabular}

*: significant $(\mathrm{p}<0.05)$

M/F: Male/female

Table 2: Laboratory findings of the patients

\begin{tabular}{llll}
\hline & Group A $(\mathrm{n}=53)$ & Group B $(\mathrm{n}=15)$ & $\mathrm{p}$ \\
\hline & $($ mean \pm SD $)$ & $($ mean \pm SD $)$ & \\
WBC & $8337.7 \pm 2873.5$ & $7826.6 \pm 2502.7$ & $\mathrm{NS}$ \\
Eos & $0.57 \pm 1.13$ & $0.42 \pm 0.34$ & $\mathrm{NS}$ \\
Alp & $119.2 \pm 84.9$ & $127.6 \pm 93.9$ & $\mathrm{NS}$ \\
GGT & $75.0 \pm 55.1$ & $139.7 \pm 155.8$ & $<0.05$ \\
& & & \\
AST & $39.9 \pm 46.0$ & $48.6 \pm 59.7$ & $\mathrm{NS}$ \\
ALT & $39.0 \pm 38.0$ & $47.4 \pm 44.2$ & $\mathrm{NS}$ \\
\hline
\end{tabular}

SD: standart deviation, WBC: white blood cell, Eos: eosinophil, ALP: alchalene phosphatase, GGT: gama glutamil transpherase, AST: Aspartate amino transpherase, ALT: Alanine aminotranspherase, NS: non significant

In group $\mathrm{B}$ biliary outflow was low then $300 \mathrm{cc} /$ day in 11 patients. These fistulas were ended spontaneously in three weeks. Four patients' fistulas were more than $300 \mathrm{cc} /$ day. At the end of three weeks endoscopic retrograd colangiopancreotography and endoscopic sphincterotomy were performed to these patients. The fistulas of three patients were stopped after the procedure. In one patient fistula was continued. The fistula was treated by fistuloenterostomy after 6 weeks in this patient. The overall hospital staying day was 5.3 days in group A and 21.2 days in group B. The difference was significant between two groups $(\mathrm{p}<0.05)$.

\section{Discussion}

In patients with HHD, the communications between the hidatid cyst cavity and the biliary tree [cysto-biliary communications ( $\mathrm{CBC})$ ] can either be occult or frank (10). The frank CBC accounts for $5 \%-17 \%$ of the cases and is easily diagnosed preoperatively period with patient story, physical examination and laboratory findings $(12,13)$. In these patients there is jaundice in the physical examination or in the story; dilated common bile duct in the ultrasound (US) or computerized tomography (CT), or magnetic resonance (MR); hiperbilirubinemia or high levels of alkalene phosphatase (ALP) or gama glutamile transpherase (GGT) in the blood samples $(12,14,15)$. Cholangitis attacks may be in some patients (16). In contrast, it is unlikely to identify occult CBCs pre-operatively and arise as external biliary fistula, biliary peritonitis or biliary abscess in postoperative period of patients $(5,14,17,18)$. Previous studies reported the development of occult CBC in $13 \%-37 \%$ of the cases (18). The diagnosis of an occult CBC can be made by the detection of a bile duct in the cyst during surgery or by means of demonstrating the bile duct on endoscopic retrograde cholangiopancreatography (ERCP) performed pre-operatively (8). In cases in which the bile duct is not observed and $\mathrm{CBC}$ could not be confirmed during surgery, $\mathrm{CBC}$ manifests itself with bile drainage through the catheters during the post-operative period $(12,15)$. In a prospective study, cysto-biliary fistulas were detected in 45 patients. As 6 patients had preoperative jaundice, the fistulas were exposed via ERCP (13.3\%). Post-operative biliary drainage was observed in 25 of the remaining 39 patients $(64.1 \%)(19)$.

Intracystic pressure is $30-80 \mathrm{~cm} \mathrm{H} 2 \mathrm{O}$, but normal biliary system pressure is $15-20 \mathrm{~cm} \mathrm{H} 2 \mathrm{O}(20,21,22)$. Flow is therefore toward the biliary system, and bile may not be present in the cavity despite occult CBC (23). Once the cyst has been drained, leakage follows because the pressure gradient is reversed, and bile flows into the residual cavity rather than through the papilla of Vater (24). That most of occult CBC cases appear as biliary leakage also supports this view $(12,25,26)$.

In the present study, frank CBC was demonstrated in $14 \%$ of the patients who underwent surgery because of HHD, and they were thus excluded from the study. As none of the patients included in the present study had signs of CBC, ERCP was not performed in any of them in the pre-operative period. CBC was noted during surgery in 19 of 68 patients 
$(27 \%)$ who participated in this study. All of them had occult $\mathrm{CBC}$ and the rate observed $(27 \%)$ was consistent with the literature.

Hepatectomy and pericystectomy are radical operations for hydatid liver cyst. Radical surgery carries a perioperative risk, but postoperative biliary leakage and recurrence are rare (27-29). The fistula is repaired in healthy tissue. Formal hepatectomy should only be performed by experienced surgeons, in specialized centres. 31 Conservative surgery, which is preferred in endemic regions, carries a high incidence of postoperative biliary leakage and local recurrence $(5,29)$. The main aims of conservative surgery are inactivation of viable elements of the parasite, evacuation of the cyst cavity and management of the residual cavity $(4,5)$. Prousalidis J offered more aggressive approaches in cysts of the upper portion of the liver, including individual thoracic and abdominal or rarely thoracoabdominal (30).

How can the risk of biliary drainage due to occult cysto-biliary fistulas be determined prior to surgery and what should be done during the surgery? ERCP is beneficial in showing the dilatation in the biliary duct and the relationship between the cyst and the bile ducts prior to surgery. However, it generally is not effective in demonstrating occult cysto-biliary fistulas because of the relationships between the very small bile ducts and high intra-cystic pressure (20). Moreover, it certainly is not possible to perform pre-operative ERCP in all patients in whom the clinical and laboratory findings do not reveal CBC. During the operation the presence of bile in the cyst fluid or the determination of an open bile duct with naked eyes in the cyst, even when the cyst fluid is clear, proves the presence of a cysto-biliary fistula. Özmen and Cosskun have suggested the use of a telescope during surgery to determine the relationship between the bile ducts and the cyst. They determined a relationship between the bile duct and the cyst in 6 of 18 patients via this simple method and sutured the fistulas. Biliary fistulas had not been seen in any of these patients post-operatively (23). If an open bile duct cannot be noted in a patient with suspected occult CBC during surgery, cysts can be filled with saline, and air can be given through the cystic channel. The other method is to inject methylene blue into the gallbladder or into the common bile duct. Air bubbles or methylene blue coming of the cyst would be helpful in detecting bile duct openings (21).

In the present study, whether or not the pre-operative laboratory findings are indicators for occult $\mathrm{CBC}$ was determined. None of the laboratory findings except GGT was useful as an indicator of occult $\mathrm{CBC}$. Owing to the fact that the bilirubin levels were between the normal ranges in all of the participants, this parameter was not included in the analyses. GGT is a biliary enzyme that is especially useful in the diagnosis of obstructive jaundice, intrahepatic cholestasis, and pancreatitis (31). GGT is more responsive to biliary obstruction than are aspartate aminotransferase (AST) and alanine aminotransferase (ALT). GGT is helpful to work up elevated alkaline phosphatase values and more specific for hepatic disease than is alkaline phosphatase (32). These two parameters were evaluated for predicting the occult $\mathrm{CBC}$ in the present study. The outcome of GGT was significantly higher in occult CBC group $(p<0.05)$. This difference can be useful for predicting of occult $\mathrm{CBC}$ in HHD preoperatively. The weakness of this study is the limited number of patients.

If occult $\mathrm{CBC}$ is found, the opening in the biliary system should be sutured with absorbable material, with or without cystic duct drainage. If no biliary opening is found, cystic duct drainage may be performed if preoperative factors predict the presence of CBC. Cavity management can then be performed by omentoplasty or external drainage, preferably with suction drainage (33). Kosmidis and his friends also covered the cut cystic cavities exposed to the peritoneum surface of the liver with fibrin glue for prevention of bile leakage. They also found that fibrin glue causes less intra-abdominal adhesions while allowing shorter haemostasis time than primary suture (34). In a study to evaluate the presumed efficacy of fibrin sealant in limiting bleeding and biliary leakage from liver residual surface after total pericystectomy for hydatid disease.by Cois A, Forty-five patients underwent total pericystectomy and liver residual surface treated with conventional techniques and fibrin sealant for control of haemorrhage and bile leakage were selected and a control group of 44 patients were carefully selected, who underwent total pericystectomy and in which fibrin sealant was not used. They found no statistical significance for the the actual role of fibrin sealant in rising efficacy on control of bleeding and biliary leakage from residual liver surface to total pericystectomy obtained with conventional haemostatic techniques (35). In another study conservative surgical procedures were performed in 23 patients (closed marsupialization with fibrin glue obliteration in 17 and drainage-marsupialization in 6), and radical surgical procedures were undertaken in 6 (pericystectomy in 5 and hemihepatectomy in 1). They found no difference for biliary leakage between two groups (36).

An external biliary fistula is the most common complication encountered after surgery for hepatic hydatid cysts (37). The rate changes between $6 \%$ and 
$28 \%$. In the present study, the rate of external biliary fistulas was $22 \%$. Although most of the external biliary fistulas close spontaneously, they may be persistent in $4 \%-27.5 \%$ of the cases (25). In the present study, low-flow fistulas ( $<300 \mathrm{ml} /$ day) were present in 11 of 15 patients with fistulas; these fistulas closed spontaneously. The remaining 4 patients had high-flow fistulas and three of them closed after ERCP, whereas one patient underwent a fistuloenterostomy. Endoscopic sphyncterectomy is performed after a 3-week waiting period in patients with low-flow fistulas or can be performed earlier in patients with high-flow fistulas $(5,38)$. Saritas et al. (6) and Dolay et al. (25) successfully treated 45 and 33 patients, respectively, with endoscopic sphyncterectomy. In the present study, we evaluated the success intraoperative suturing of the bile duct statistically and find out that intra-cystic sutures cannot prevent the development of fistulas significantly.

Biliary fistula develops when the postoperative leak is able to drain; if it cannot, biliary peritonitis and biliary abscess develop (5). Occult CBC significantly increases the complication rate (15). In our study, the complication rate was $7 \%$ in patients without biliary leakage, and $43 \%$ in those with leakage, which is consistent with the findings of other studies $(5,15,26)$. On the other hand the hospital stay length was 5-7 days in patients without biliary leakage and 14-17 days in the patients with biliary leakage $(16,38)$.

In conclusion, GGT as a labaratory test for predicting occult $\mathrm{CBC}$ preoperatively have been shown to be useful in the clinical practice. However, larger prospective studies are needed on this subject. Occult cysto-biliary fistulas can only be exposed during surgery when suspected by a surgeon. If occult CBC is found, the opening in the biliary system should be sutured with absorbable material, with or without cystic duct drainage. If no biliary opening is found, cystic duct drainage may be performed if preoperative factors predict the presence of $\mathrm{CBC}$. Also use of fibrin glue seems to be effecient for occult CBC. We believe that well planned controlled prospective studies could give the needed further elements to precisely evaluate the role of fibrin sealant in the surgical treatment of hydatid disease of the liver. As the development of external biliary fistulas increases the morbidity and the hospitalization period, novel surgical methods to prevent the development of bile fistulas are required in such patients.

\section{Author contributions}

Atahan $\mathrm{K}$ and Küpeli $\mathrm{H}$ contributed equally to this work; Atahan K, Küpeli H, Gür S designed research; Atahan K, Deniz M and Çökmez A performed research; Atahan K, Gür S and Tarcan E contributed new reagents/analytic tools; Çökmez A and Tarcan E analyzed data; Atahan K and Gür S wrote the paper.

\section{Conflict of Interest}

The authors have declared that no conflict of interest exists.

\section{References}

1. Sayek I, Yalin R, Sanac Y. Surgical treatment of hydatid disease of the liver. Arch Surg 1980,115:847-50.

2. Ulualp KM, Aydemir I, Senturk H, et al. Management of intrabiliary rupture of hydatid cyst of the liver. World J Surg 1995, 19:720-4.

3. Balik AA, Basoglu M, Çelebi F, et al. Surgical treatment of hydatid disease of theliver. Review of 304 cases. Arch Surg 1999, 134:166-9.

4. Erdem E, Nessar M, Sungurtekin U, et al. The management of hydatid cyst: review of 94 cases. J Hepatobiliary Pancreat Surg 1998,5:179-83.

5. Skroubis G, Vagianos C, Polydorou A, et al. Significance of bile leaks complicating conservative surgery for liver hydatidosis. World J Surg 2002, 26:704-8.

6. Saritas U, Parlak E, Akoglu M, et al. Effectiveness of endoscopic treatment modalities in complicated hepatic hydatid disease after surgical intervention. Endoscopy 2001, 33:858-63.

7. Koksal N, Muftuoglu T, Gunerhan Y, et al. Management of intrabiliary ruptured hydatid disease of the liver. Hepatogastroenterology 2001, 48:1094-6.

8. Kayaalp C, Bostanci B, Yol S, et al. Distribution of hydatid cysts into the liver with reference to cystobiliary communications and cavity-related complications. Am J Surg 2003, 185:175-9.

9. Bedirli A, Sakrak O, Sozuer EM, et al. Surgical management of spontaneous intrabiliary rupture of hydatid liver cysts. Surg Today 2002, 32:594-7.

10. Hankins JR. Management of complicated hydatid cysts. Ann Surg 1963, 158:1020-34.

11. Kayaalp C, Bzeizi K, Demirbag AE, et al. Biliary complications after hydatid liver surgery. Incidence and risk factors. J Gastrointest Surg 2002, 6:706-12.

12. Kornaros SE, Aboul-Nour T. Frank intrabiliary rupture of hydatid hepatic cyst: diagnosis and treatment. J Am Coll Surg 1996, 183:466-70.

13. Akkiz H, Akinoglu A, Çolakoglu S, et al. Endoscopic management of biliary hydatid disease. Can J Surg 1996, 39:287-92.

14. Alper A, Ariogul O, Emre A, et al. Choledochoduodenostomy for intrabiliary rupture of hydatid cysts of liver. Br J Surg 1987, 74:243-5.

15. Lygidakis NJ. Diagnosis and treatment of intrabiliary rupture of hydatid cyst of the liver. Arch Surg 1983, 118:1186-9.

16. Ovnat A, Peiser J, Avinoah E, et al. Acute cholangitis caused by ruptured hydatid cyst. Surgery 1984, 95:497-500.

17. Magistrelli $P$, Masetti R, Coppola R, et al. Surgical treatment of hydatid disease of the liver. A 20-year experience. Arch Surg 1991, 126:518-23.

18. Becker K, Frieling T, Saleh A, et al. Resolution of hydatid liver cyst by spontaneous rupture into the biliary tract. J Hepatol 1997, 26:1408-12.

19. Marti-Bonmati L, Menor F, Ballesta A. Hydatid cyst of the liver: rupture into the biliary tree. AJR Am J Roentgenol 1988, 150:1051-3.

20. Ozaslan E, Bayraktar Y. Endoscopic therapy in the management of hepatobiliary hydatid disease. J Clin Gastroenterol 2002, 35:160-74. 
21. Milicevic H. Hydatid disease. In: Blumgart L, Fong Y, editors. Surgery of the liver and biliary tract; 2 nd ed. Philadelphia: WB Saunders Company, 2000:1167-204.

22. Yalin R, Aktan AÖ, Yegen C, et al. Significance of intracystic pressure in abdominal hydatid disease. Br J Surg 1992, 79:1182-3.

23. Özmen M, Coskun F. New technique for finding the ruptured bile duct into the liver cyst: scope in the cave technique. Surg Laparosc Endosc Percutan Tech 2002, 12:187-9.

24. Vignote ML, Mino G, de la Mata M, et al. Endoscopic sphincterotomy in hepatic hydatid disease open to the biliary tree. Br J Surg 1990, 77:30-1.

25. Dolay K, Akçakaya A, Soybir G, et al. Endoscopic sphincterotomy in the management of postoperative biliary fistula. A complication of hepatic hydatid disease. Surg Endosc 2002, 16:985-8.

26. Zaouche A, Haouet K, Jounini M, et al. Management of liver hydatid cysts with a large biliocystic fistula: multicenter retrospective study. World J Surg 2001, 25:28-9.

27. Giordano G, Angrisano A, Palazzo P, et al. Surgical treatment of hydatid cyst of the liver: pericystectomy or resection. Personal experience. Int J Surg Sci 1999;6: 113-7.

28. Moreno Gonzalez E, Rico Selas P, Martinez B, et al. Results of surgical treatment of hepatic hydatidosis: current therapeutic modifications. World J Surg 1991;15:254-63.

29. Kayaalp C, Sengul N, Akoglu M. Importance of cyst content in hydatid liver surgery. Arch Surg 2002;137:159-63.

30. Prousalidis J, Kosmidis C, Kapoutzis K, et al. Intrabiliary rupture of hydatid cysts of the liver. Am J Surg. 2009;197(2):193-8. 30. Stein TA, Burns GP, and Wise L, "Diagnostic Value of Liver Function Tests in Bile Duct Obstruction," J Surg Res 1989, 46(3):226-9.

31. Singh M.K., et al. Gamma-Glutamyl Transpeptidase (GGT) As A Marker In Obstructive Jaundice . The Internet Journal of Surgery 2007, 9:2

32. Demircan O, Baymuş M, Seydaoğlu G, et al. Occult cystobiliary communication presenting as postoperative biliary leakage after hydatid liver surgery: Are there significant preoperative clinical predictors? J Can Chir 2006, 49(3): 177-184.

33. Kosmidis C, Efthimiadis C, Anthimidis G, et al. Combined left hepatectomy with fenestration and using a harmonic scalpel, fibrin glue and closed suction drainage to prevent bile leakage and ascites in the management of symptomatic polycystic liver disease: a case report. Journal of Medical Case Reports 2009, 3:7442

34. Cois A, Iasillo G, Nardello O, et al. Human fibrin glue in the treatment of residuel parenchymal surface after total pericystectomy for hepatic echinococcus. Ann Ital Chir 1997;68(5):701-6.

35. Hofstetter C, Seqovia E, Vara-Thorbeck R. Treatment of uncomplicated hydatid cyst of the liver by closed marsupialization and fibrin glue obliteration. World J Surg 2004 Feb;28(2):173-8.

36. Dadoukis J, Prousalidis J, Botsios D, et al. External biliary fistula. HPB Surg 1998, 10:375-7.

37. Gharbi HA, Hassine W, Brauner MW, et al. Ultrasound examination of the hydatic liver. Radiology 1981, 139:459-63.

38. Atli M, Kama NA, Yuksek YN, et al. Intrabiliary rupture of a hepatic hydatid cyst associated clinical factors and proper management. Arch Surg 2001, 136:1249-55. 